\title{
Fabrication of a Multimode Interference Device in a Low-Loss Flat-Fiber Platform Using Physical Micromachining Technique
}

\author{
Sumiaty Ambran, Member, OSA, Christopher Holmes, James C. Gates, Andrew S. Webb, Student Member, OSA,
} Lewis G. Carpenter, Faisal Rafiq Mahamd Adikan, Peter G. R. Smith, and Jayanta K. Sahu, Member, OSA

\begin{abstract}
A physical micromachining technique is demonstrated in a low-loss flat-fiber substrate to fabricate a multimode interference (MMI) device. The flat-fiber substrate is a low-index-contrast material; however, by making use of two physically micromachined trenches, lateral confinement is achieved providing high index contrast for the MMI region. A $1 \times 3 \mathrm{MMI}$ device exhibiting $1.89 \mathrm{~dB}$ of excess loss has been demonstrated.
\end{abstract}

Index Terms-Air trenches, flat fiber, multimode interference (MMI).

\section{INTRODUCTION}

$\mathbf{M}$ ULTIMODE INTERFERENCE (MMI) devices have attracted interest in integrated optics owing to their widespread benefits such as small device dimensions and a broad spectral bandwidth [1]. MMI devices have been successfully demonstrated in a wide range of applications, particularly for power splitting and combining purposes. Recently, they have been proposed for more active applications, including optical sensing [2] and switching [3]. The device structure has been extensively demonstrated in both low- and high-index-contrast materials, for example, silica-on-silicon [4], polymer [5], and silicon-on-insulator [6] platforms; yet, most of these materials are either relatively expensive, have low index contrast, exhibit high loss, or have complex fabrication processes. The study reported in this paper uses a novel flat fiber [7], [8] to fabricate an MMI structure. This platform has the benefits of being relatively inexpensive, yielding a low insertion loss and being mechanically flexible compared to traditional planar platforms.

Manuscript received January 16, 2012; revised March 21, 2012; accepted May 07, 2012. Date of publication May 15, 2012; date of current version August 17, 2012. This work was supported in part by the University of Southampton, Ministry of Higher Education High Impact Research Grant (Integrated Photonics for Biosensors), Malaysia, and the University of Technology Malaysia.

$\mathrm{S}$. Ambran is with the Optoelectronics Research Center, University of Southampton, SO17 1BJ, U.K., and also with the Malaysia-Japan International Institute of Technology, University Technology of Malaysia, Kuala Lumpur, Malaysia (e-mail: sa4g09@orc.soton.ac.uk).

C. Holmes, J. C. Gates, A. S. Webb, L. G. Carpenter, P. G. R. Smith, and J. K. Sahu are with the Optoelectronics Research Center, University of Southampton, SO17 1BJ, U.K. (e-mail: chh@orc.soton.ac.uk; jcg@orc.soton.ac.uk; asw@orc. soton.ac.uk; lc906@orc.soton.ac.uk; pgrs@orc.soton.ac.uk; jks@orc.soton.ac. uk).

F. R. M. Adikan is with the Department of Electrical Engineering, University of Malaya, Kuala Lumpur, Malaysia (e-mail: Rafiq@um.edu.my).

Color versions of one or more of the figures in this paper are available online at http://ieeexplore.ieee.org.

Digital Object Identifier 10.1109/JLT.2012.2199465

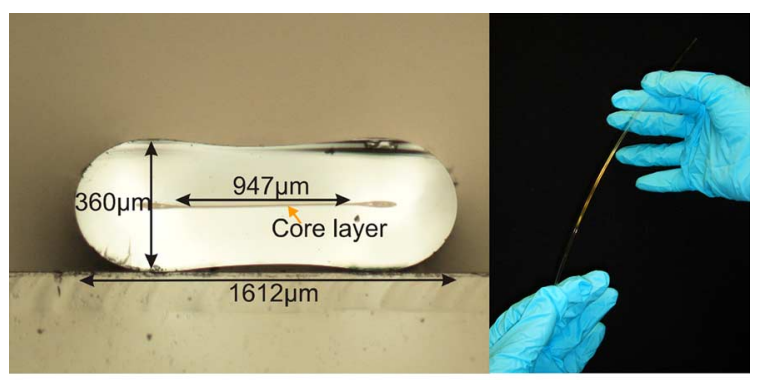

(a)

(b)

Fig. 1. (a) Cross section and (b) bending of the flat fiber. The index contrast of the germanium-doped core and the cladding of the flat fiber is 0.0183 .

In addition, the physical micromachining technique reported provides the advantages of high-index-contrast design.

Flat fiber has a similar fabrication technique as standard optical fiber, using modified chemical vapor deposition (MCVD) and fiber drawing processes to lead to a low-cost planar substrate [7]. The process differs from the standard fiber fabrication during the collapse of the preform. This occurs at the fiber drawing stage, where a vacuum is applied to flatten the preform into a planar geometry. Fig. 1(a) shows the cross section and (b) the bending of a length of flat fiber showing its geometry and flexibility. In the context of conventional MMI devices, the flat-fiber platform would typically be considered a low-index-contrast material. Such low-index-contrast systems lead to lower optical confinement and, thus, reduced performance of the resulting MMI device [9]. However, it does have the benefit of providing low coupling loss, with standard silica optical fibers. This is due to better mode matching, compared to that of high-index-contrast platforms, and a comparable refractive index to fiber unlike materials such as silicon.

In this study, we combine the benefits associated with lowand high-index-contrast materials. The low index contrast is defined vertically, provided by a germanium (Ge)-doped core layer ( $n=1.4623)$ sandwiched by a top and bottom cladding layer ( $n=1.444)$ of the flat fiber. In our devices, the high index contrast is formed horizontally between air trenches and the core layer as illustrated in Fig. 2.

In the past, various techniques have been used to form highindex-contrast MMI structures. For example, one of the reported techniques used metal-clad sidewalls, where the waveguide surface was surrounded by a thin metal layer. This acts to increase the index contrast of the MMI device. However, this technique is 


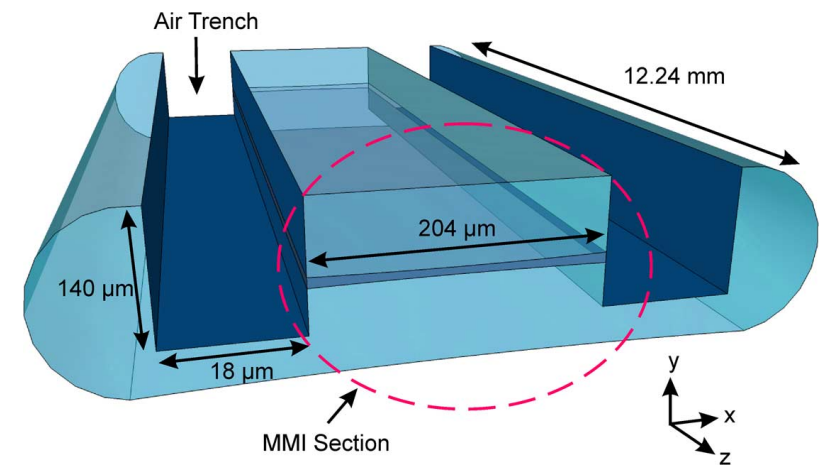

Fig. 2. 3-D schematic diagram of the micromachined trenches in the flat-fiber substrate. Two trenches were cut parallel to the MMI section to define the region of light confinements.

limited to operating with one polarization only as the TE cannot be supported within the waveguide [10]. The concept presented in this paper uses a similar concept to previous work [9], [11], where air trenches are used to define a large index contrast of the MMI section. In general, such an approach affords strong lateral confinement, and with it, good imaging performance [12] and coupling efficiency [9].

Several fabrication techniques have previously been reported to produce such high-index lateral confinement of the MMI device. Photolithography followed by etching is the conventional fabrication technique and has been widely used in many integrated optical applications [9], [13]. However, these cleanroom techniques can be time consuming, and require several stages to complete a device. This paper presents an alternative fabrication technique employing physical micromachining of the flat-fiber substrate to form the MMI section using air trenches. The novelty behind this study is that it combines physical micromachining with a low-cost flat-fiber fabrication platform. Furthermore, with the flat-fiber format, it is difficult to use lithography and etching simply because of the difficulty of spinning photoresists on such a geometry. The technique used here offers a simple and straightforward fabrication approach where a diamond-blade dicing saw is used to physically machine a pair of trenches, as illustrated in Fig. 2. This micromachining method has been previously used to fabricate micro-optical mechanical systems [14].

This paper is structured first to detail the theoretical modeling of the MMI device. The concept of the micromachined trenches is described in Section II. The technique used to fabricate the MMI device is explained in Section III. In Section IV, we elaborate on the characterization setup used to measure the performance of the device. Finally, Section V contains experimental results and a discussion and Section VI concludes this paper.

\section{DESIGN AND CONCEPT}

Self-imaging is a key concept for MMI device operation where a single transverse optical mode at the input of the device can be reproduced as a single or multimode image at certain positions along it. The self-imaging operation of an MMI device primarily depends on three parameters, the effective width $W_{\text {eff }}$, the effective refractive index $n_{\text {eff }}$, and the

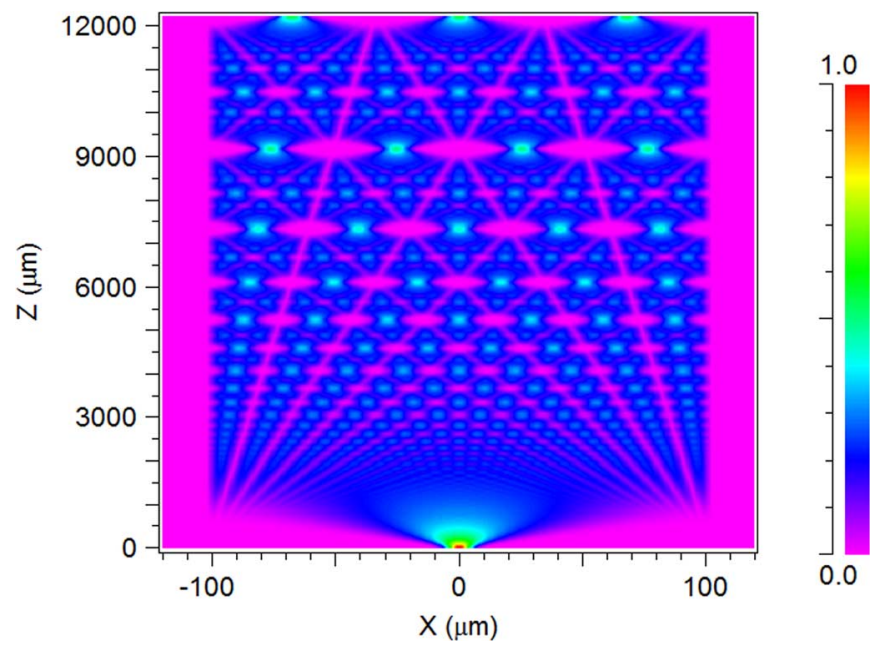

Fig. 3. BeamPROP modeling of the propagation light within the MMI section. Single-mode light is launched into the center of the MMI section, and three output spots are produced at the output section. The wavelength of $1652 \mathrm{~nm}$ was implemented in the modeling.

operation wavelength $\lambda$, where the length of the device $L_{\mathrm{MMI}}$ is given by (1) [15]

$$
L_{\mathrm{MMI}}=\frac{n_{\mathrm{eff}}\left(W_{\mathrm{eff}}\right)^{2}}{\lambda} .
$$

Fig. 2 shows the 3-D schematic diagram of the MMI device, where two micromachined trenches were physically machined to form an MMI section. These trenches form a high-index interface and thus give lateral confinement for both sides of the MMI region. The vertical confinement is achieved by the flat-fiber core layer having a higher refractive index than the cladding and is due to germanium doping.

As way of demonstration, we designed an MMI device as a $1 \times 3$ splitter, where the trenches confine the light to regenerate the input mode at three individual locations at the output facet. The MMI structure has been modeled using commercial BeamPROP software from the Rsoft design group in order to predict the dimensions of the MMI structure as well as determine the effect of certain parameters. In the modeled system, the slab waveguide mode, in the $y$-axis, had an effective index of 1.4592 at $1652 \mathrm{~nm}$; this reduces the problem to two dimensions [15]. A Gaussian mode was selected as the launched light parameter with a mode-field diameter (MFD) of $10.5 \mu \mathrm{m}$ to represent the polarization maintaining (PM) fiber used in the measurement setup. The light propagation pattern throughout the MMI waveguide is shown in Fig. 3 for the specific case of a $1 \times 3 \mathrm{MMI}$ device.

\section{MMI DEVICE FABRICATION}

The $1 \times 3$ MMI splitter device was fabricated in the flat-fiber platform. The core layer thickness of this particular fiber was $6.0 \mu \mathrm{m}$ and provides single vertical mode confinement. The refractive indices of the core and cladding layers are 1.4623 and 1.444, respectively. The cladding index value is known for the Suprasil F300 tube material, and the appropriate core index is inferred from a FIMMWAVE simulation that yields the core layer refractive index of 1.4623 at $1652 \mathrm{~nm}$. This value is 


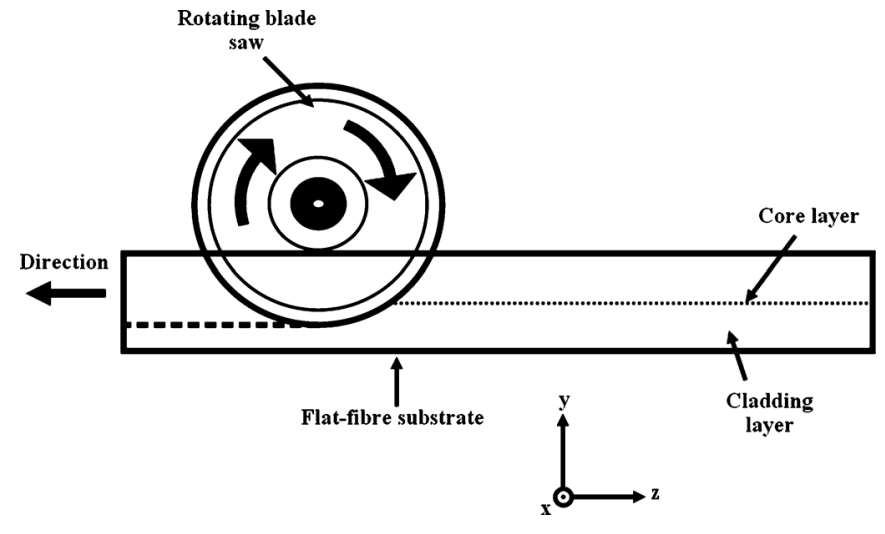

Fig. 4. Schematic diagram illustrating how the dicing machine is used to fabricate the MMI air trenches. The substrate is translated in the $z$-direction where the dicing is made to cut the core layer section laterally.

in-line with the expected value for the core glass based upon the known doping parameters of the MCVD fiber process. Two trenches were physically cut using an ultraprecision dicing saw with a 5000 grit nickel-bonded diamond blade as depicted in Fig. 4. The overcladding of the flat fiber was removed via lapping to within about $100 \mu \mathrm{m}$ of the core layer. This reduced the total depth of the trench cuts and allowed sawing into the core layer using the blades available. The depth and the width of the trenches were 140 and $18 \mu \mathrm{m}$, respectively. These saw cuts define the width of the MMI device and were measured postfabrication as $204 \mu \mathrm{m}$. For this proof-of-principle device, an MMI width of $204 \mu \mathrm{m}$ was chosen to provide sufficient output channel spacing to assist in the analysis of the output via standard fibers. A future optimized device would have to consider the design constraints associated with MMI width minimization [16] and the output coupling. The length of the device was predicted using BeamPROP modeling, which was $12.54 \mathrm{~mm}$ long. The end facet and hence the length of the device was machined by the same dicing saw used to machine the trenches and hence had similar fabrication errors. These dimensions are optimized for the $1 \times 3$ splitting of laser light at $1652 \mathrm{~nm}$. The operating wavelength is dominated by the precision of the micromachining, the width of the MMI being the most sensitive dimension. The micromachining system used in this study has a blade positional error of $2 \mu \mathrm{m}$ and a blade width error of $2 \mu \mathrm{m}$. In commercial dicing machines, these tolerances can be greatly reduced to submicrometer dimensions. Fig. 5(a) shows the end facet of the MMI section after micromachining, the trenches run parallel to the MMI section. A top view of the scanning electron microscope (SEM) image is depicted in Fig. 5(b). Although this does not provide a direct measurement of the width of the core layer, it shows that the micromachining technique yields very small wedge variation of the MMI section. Analysis via imaging white light interferometry (ZeScope made by Zemetrics) indicates width variation of less than $0.5 \mu \mathrm{m}$ over the $12.24 \mathrm{~mm}$ length of the device.

\section{Characterization}

To observe the modal output from the device light, a tunable laser source was launched into the middle of the MMI section via a PM fiber as depicted in Fig. 6. The output mode pat-

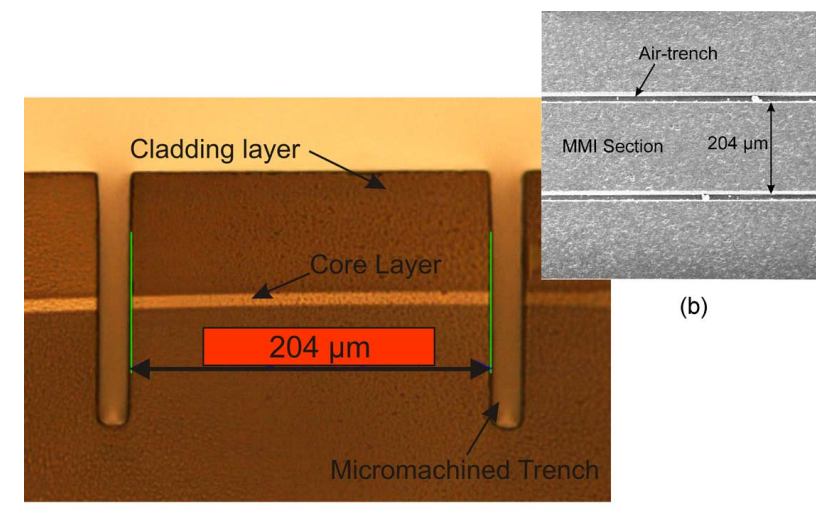

(a)

Fig. 5. (a) Microscope image of the end facet of the MMI section and (b) Top view of the SEM image showing the parallel characteristics along the MMI length. Two trenches were cut allowing the core layer being exposed to the air, providing a high index contrast between the refractive index of the core layer and the air trenches.

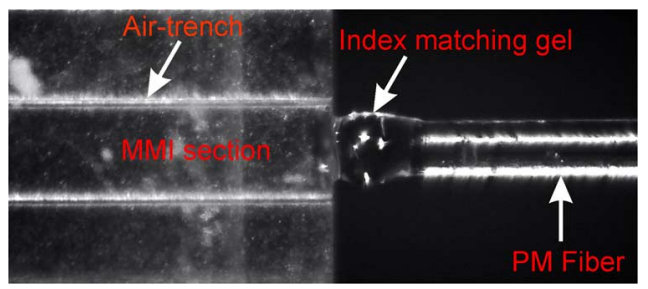

Fig. 6. Coupling image between the MMI section and the PM fiber

tern was observed using a near infrared (NIR) camera. Due to the restricted range of the tunable laser, this was measured at $1641 \mathrm{~nm}$ which is close to the optimal operating wavelength of $1652 \mathrm{~nm}$. A 20x objective was located between the end facet of the MMI device and the NIR camera. Due to the low dynamic range of the NIR camera, an alternative method to record the device operation used a fiber that was directly butt coupled to the output of the MMI and translated to sample the optical intensity at the output facet. A PM fiber was used in order to maintain the light polarization, and the coupled power was measured using a power meter. The measurements were made using TM polarization.

To investigate the wavelength dependence of the device, a spontaneous LED broadband light source (1610-1675 nm) was coupled into the device and the spectrum from each output was collected with an optical fiber. The collected light was analyzed using an optical spectrum analyzer (ANDO AQ 6317B).

\section{RESUlTS AND Discussion}

The modal image of the three output spots recorded by the NIR camera is shown in Fig. 7(a). The simulated and experimental results of the output intensity distribution can be seen in Fig. 7(b). The simulation result has been convoluted with a Gaussian mode profile with MFD of $10.5 \mu \mathrm{m}$ to represent the PM fiber that is used for scanning the MMI output facet. Modeling of the spectral dependence was also implemented to observe the output peak power as a function of wavelength.

The insertion loss for each three spots was measured, as illustrated in Fig. 8. It can be clearly observed that the output 


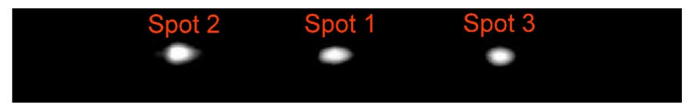

(a)

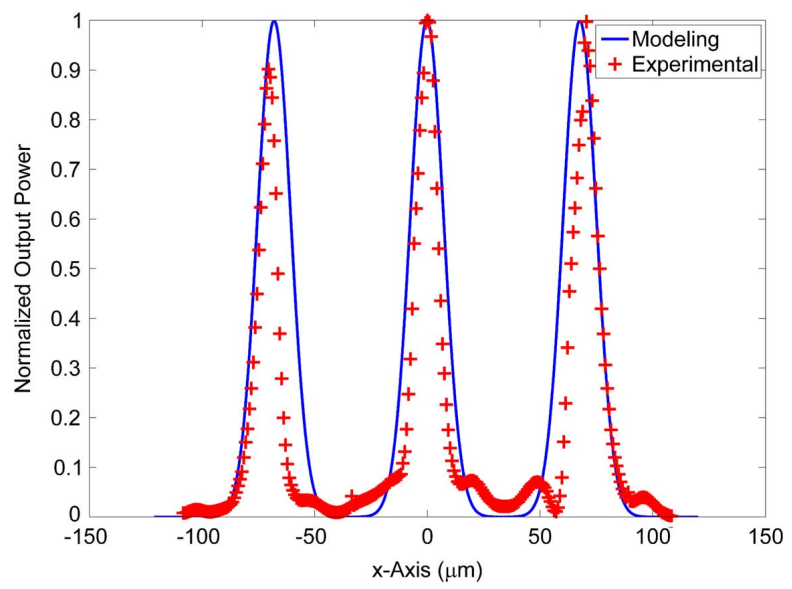

(b)

Fig. 7. (a) Modal image of the $1 \times 3$ MMI device recorded by the NIR camera at $1641 \mathrm{~nm}$ and (b) quantitative optical output of the modal image via scanning measurement technique at the end facet of the MMI region using the PM fiber. The scanning is implemented horizontally along the width of the MMI section.

powers from each spot are strongly dependent on the wavelength of the light coupled into the MMI. Also, the optimum wavelength, i.e., lowest insertion loss, of the three spots is very similar. The output from spot 3 shows a small oscillation that we believe is due to a slight cavity formed between the MMI device and fiber connection. From the measurement, it shows that the optimum wavelength for the device was $1652 \mathrm{~nm}$ with the total excess loss of $1.89 \mathrm{~dB}$. The total excess loss of the device was calculated by taking the ratio between the launched power into the sample and the sum of the three peak outputs. It is worth noting that the most equal splitting ratio occurs at 1620 $\mathrm{nm}$ but this is outside the $1 \mathrm{~dB}$ bandwidth as described here. From Fig. 8, we find that the bandwidth of minimum excess loss, less than $1 \mathrm{~dB}$ increase, spans from 1636 to $1673 \mathrm{~nm}$ corresponding to a width of $37 \mathrm{~nm}$. This can be compared to recent reports of photonic-crystal-based beam splitters demonstrating a $1 \mathrm{~dB}$ bandwidth of $11 \mathrm{~nm}$ in the $C$-band [17]. Over this spectral bandwidth, the uniformity of the outputs is $1.24 \mathrm{~dB}$ which is comparable to current commercially available devices. Fig. 9 shows the normalized transmission from the experiment data compared to the BPM model, as a function of wavelength. The results agree within the wavelength range of 1635-1660 nm. Outside this spectral range, a small disagreement between the modeling and experimental result can be seen from the graph. The discrepancy between the experimental data and the model is due to the dispersion, as this was not been taken into consideration when modeling the MMI device.

There are three main contributions associated with the total excess loss of the experimental result. They are propagation loss, coupling loss, and scatter loss. In a preliminary experiment, the propagation loss of the flat fiber was measured via two different measurement methods at $1550 \mathrm{~nm}$ wavelength. These were a Bragg grating loss measurement technique [18] and a measurement using an optical backscattered reflectometer

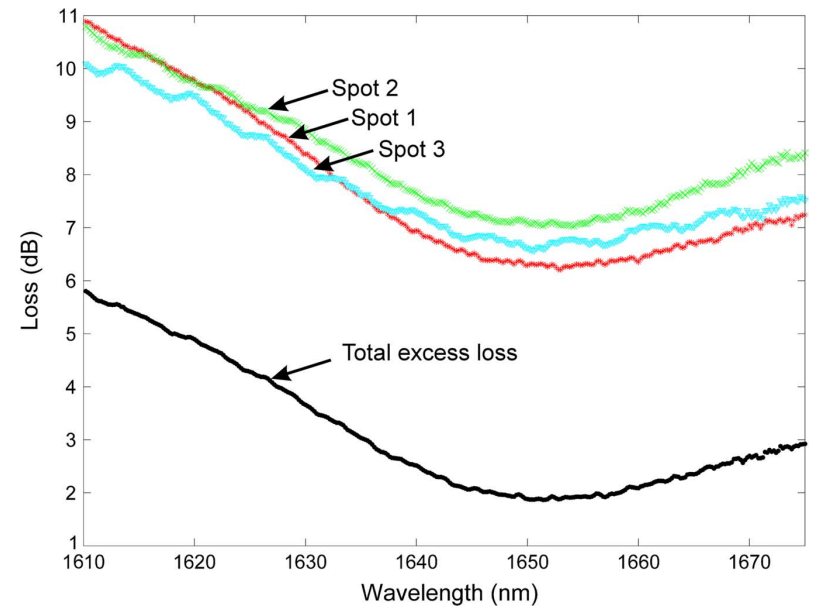

Fig. 8. Insertion loss from each of the three output spots against the wavelength. The total excess loss is also calculated by summing all three output powers at each respective wavelength.

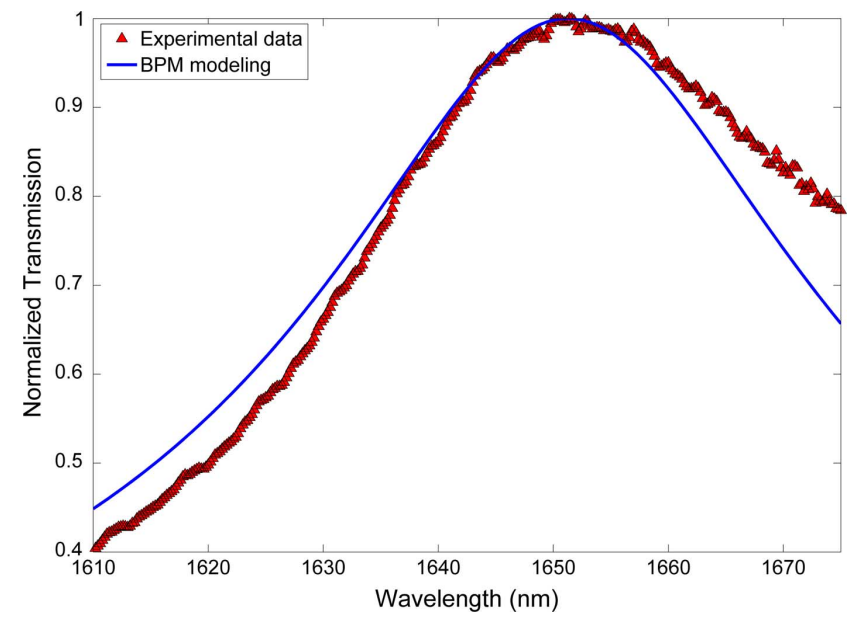

Fig. 9. Normalized transmission from the modeling represented as solid line, showing good agreement to the experimental data. The optimum wavelength of the maximum point is $1652 \mathrm{~nm}$.

(OBR) model 4400 from Luna technology system. The measured loss of the flat fiber was $0.12 \pm 0.02 \mathrm{~dB} / \mathrm{cm}$ for the Bragg grating loss measurement and $0.09 \pm 0.01 \mathrm{~dB} / \mathrm{cm}$ for the OBR system. These two different measurements show good agreement and also concur with that observed with previous samples [7]. Even though the number provided here is the propagation loss specifically for UV-written channel waveguides, it is used as an estimation of the anticipated upper bound for the intrinsic propagation loss within the flat-fiber material. This estimation of propagation loss includes the material loss and scatter loss from cladding and core layers. It does not include the scatter loss from the trench sidewalls, which is discussed later.

To estimate coupling losses, an overlap integral method has been implemented, whereby the fiber was scanned across the output facet using translation stages. The output mode of the MMI device was measured vertically and horizontally in order to estimate an MFD for each axis. Separately, the MFD of the PM fiber was measured by scanning the PM fiber against an identical piece of PM fiber. Both output modes were fitted with a Gaussian mode. Then, the measured MFD of the MMI output 
mode and the PM fiber was overlapped. The outcome from the overlap integral showed that the coupling loss was $\sim 0.6$ $\mathrm{dB}$ per facet providing $\sim 1.2 \mathrm{~dB}$ coupling loss in total for the input and output fiber coupling to the MMI device. This coupling loss could be reduced by optimizing the planar layer core parameters.

Another anticipated source of losses is scattering from the sidewalls. This is difficult to investigate directly; however, one approach is to put index matching oil into the trenches. This reduces the scattering by lowering index contrast - but also changes the MMI properties making it difficult to make quantitative measurements. However, we believe the scattering loss of the device is small as no significant effect in term of the device performance was observed after an index oil of 1.33 was placed within the trenches.

The excess loss measured here $(1.89 \mathrm{~dB})$ is still higher than expected from the modeling results. Removing the known losses in the system, i.e., the propagation loss and the fiber coupling losses, an additional loss of $0.57 \mathrm{~dB}$ remains. This higher loss is expected to be associated with factors that the modeling does not account for, such as the scattering loss due to the surface roughness and a possible lack of verticality of the trench sidewalls. This magnitude of loss is comparable to other reported work using conventional fabrication techniques [19]-[21].

\section{CONCLUSION}

We have demonstrated for the first time a $1 \times 3$ MMI device fabricated by physical micromachining channels in a low-loss flat-fiber substrate. Although the flat-fiber substrate is a low-index-contrast platform, by using two air trenches, a high-index lateral contrast can be produced to achieve better output imaging. The MMI device has $1.89 \mathrm{~dB}$ excess loss, which was attributed to fabrication imperfection and scatter loss. Such components could be integrated into longer flat-fiber lengths and combined with previously published UV writing technology [7] for splitting and combining operations.

\section{REFERENCES}

[1] L. B. Soldano and E. C. M. Penning, "Optical multi-mode interference devices based on self-imaging: Principles and application," J. Lightw. Technol., vol. 13, no. 4, pp. 615-627, Apr. 1995.

[2] M. Mayeh, J. Viegas, P. Srinivasan, P. Marques, J. L. Santos, E. G. Johnson, and F. Farahi, "Design and fabrication of slotted multimode interference devices for chemical and biological sensing," J. Sensor, pp. 470175-1-470175-11, 2009.

[3] Z. Jin, C. J. Kaalund, and G. Peng, "Novel approach to design high performance large-port-count switches in low-index-contrast materials based on cascaded multimode interference couplers," IEEE J. Quantum Electron., vol. 41, no. 12, pp. 1548-1551, Dec. 2005.

[4] C. Holmes, H. E. Major, J. C. Gates, C. B. E. Gawith, and P. G. R. Smith, "Period adapted Bragg mirror multimode interference device," in Proc. Conf. Lasers Electro-Opt./Quantum Electron. Laser Sci. Conf., 2009, pp. 1-2.

[5] M. H. Ibrahim, N. M. Kassim, A. B. Mohammad, M. K. Chin, and S. Y. Lee, "Multimode interference optical splitter based on photodefinable benzocyclobutene (BCB 4024-40) polymer," Opt. Eng., vol. 46, no. 1, pp. 013401-1-013401-4, 2007.

[6] H. Chen and D. T. K. Tong, "Two-dimensional symmetric multimode interferences in silicon square waveguide," IEEE Photon. Technol. Lett., vol. 17, no. 4, pp. 801-803, Apr. 2005.

[7] A. S. Webb, F. R. M. Adikan, J. K. Sahu, R. J. Standish, C. B. E. Gawith, J. C. Gates, P. G. R. Smith, and D. N. Payne, "MCVD planar substrates for UV-written waveguide devices," Electron. Lett., vol. 43, no. 9, pp. 517-519, 2007.
[8] F. R. M. Adikan, S. Sandoghchi, W. Chong, R. Simpson, M. Mahdi, A. Webb, J. Gates, and C. Holmes, "Direct UV written optical waveguides in flexible glass flat fibre chips," IEEE J. Sel. Topics Quantum Electron. , to be published.

[9] X. Wang and R. T. Chen, "Image enhanced polymer-based multimode interference coupler covering $\mathrm{C}$ and $\mathrm{L}$ bands using deeply etched air trenches," Appl. Phys. Lett., vol. 90, pp. 111106-1-111106-3, 2007.

[10] J. Z. Huang, M. H. Hu, J. Fujita, R. Scarmozzino, and R. M. Osgood, "High-performance metal-clad multimode interference devices for low-index-contrast material systems," IEEE Photon. Technol. Lett., vol. 10, no. 4, pp. 561-563, Apr. 1998.

[11] C. J. Kaalund and Z. Jin, "Novel multimode interference devices for low index contrast materials systems featuring deeply etched air trenches," Opt. Commun., vol. 250, pp. 292-296, 2005.

[12] M. K. Chin, C. W. Lee, S. Y. Lee, and S. Darmawan, "High-index-contrast waveguides and devices," Appl. Opt., vol. 44, no. 15, pp. 3077-3086, 2005.

[13] Y. Ma, S. P. L. Wang, and S. T. Ho, "Ultracompact multimode interference 3-dB coupler with strong lateral confinement by deep dry etching," IEEE Photon. Technol. Lett., vol. 12, no. 5, pp. 492-494, May 2000.

[14] L. G. Carpenter, C. Holmes, H. L. Rogers, P. G. R. Smith, and J. C. Gates, "Integrated optic glass microcantilevers with Bragg grating interrogation," Opt. Exp., vol. 18, no. 22, pp. 23296-23301, 2010.

[15] K. Okamoto, "Fundamentals of Optical Waveguides," in Multimode Interference (MMI) Device, 2nd ed. New York: Academic, 2006, pp. $46-55$.

[16] A. Hosseini, H. Subbaraman, D. Kwong, Y. Zhang, and R. T. Chen, "Optimum access waveguide width for $1 \times \mathrm{N}$ multimode interference couplers on silicon nanomembrane," Opt. Lett., vol. 35, no. 17, pp. 2864-2866, 2010

[17] M. Zhang, R. Malureanu, A. C. Kruger, and M. Kristensen, " $1 \times 3$ beam splitter for TE polarization based on self-imaging phenomena in photonic crystal waveguides," Opt. Exp., vol. 18, no. 14, pp. 14944-14949, 2010.

[18] H. L. Rogers, S. Ambran, C. Holmes, P. G. R. Smith, and J. C. Gates, "In situ loss measurement of direct UV written waveguides using integrated Bragg gratings," Opt. Lett., vol. 35, no. 17, pp. 2849-2851, 2010.

[19] M. Bruendel and D. G. Rabus, " $1 \times 2$ and $1 \times 3$ multimode interference couplers fabricated by hot embossing and DUV-induced modification of polymers," in Proc. 19th Annu. Meet. IEEE Conf. Lasers ElectroOpt. Soc., 2006, pp. 326-327.

[20] P. A. Besse, E. Gini, M. Bachmann, and H. Melchior, "New $2 \times 2$ and $1 \times 3$ multimode interference couplers with free selection of power splitting ratios," J. Lightw. Technol., vol. 14, no. 10, pp. 2286-2293, Oct. 1996.

[21] J. M. Hong, H. H. Ryu, S. Y. Park, J. W. Jeong, S. G. Lee, E.-H. Lee, S.-G. Park, D. Woo, S. Kim, and O. Beom-Hoan, "Design and fabrication of a significantly shortened multimode interference coupler for polarization splitter application," IEEE Photon. Technol. Lett., vol. 15, no. 1, pp. 72-74, Jan. 2003.

Sumiaty Ambran received the B.S. degree in electronics telecommunication from the University Technology of Malaysia, Johor, Malaysia, in 2005, and the Master's degree from the University of MARA, Selangor, Malaysia, in 2008. She is currently working toward the Ph.D. degree in Silica fiber Fabrication and Planar Optical Materials Group, Optoelectronics Research Centre, University of Southampton, Southampton, U.K.

She is currently involved in the development of UV-written flat-fiber substrate for planar optical devices. She is also with the Malaysia-Japan International Institute of Technology, University Technology of Malaysia, Johor. Her research interests include rare-earth-doped optical devices for laser and amplifier application.

Ms. Ambran is a member of the Optical Society of America.

Christopher Holmes received the M.S. degree (first-class hons.) in physics from Warwick University, Coventry, U.K., in 2004, and the Ph.D. degree in direct UV-written devices from the University of Southampton, Southampton, U.K., in 2009.

$\mathrm{He}$ is currently a Research Fellow at the University of Southampton, where he is developing new technologies in integrated optics. During his Ph.D., he received a CASE scholarship from the photonics company Stratophase Ltd., for his research on UV-written devices for detection applications. He is currently developing micromachined integrated optical devices for telecommunication and detection applications. 
James C. Gates received the M.Phys. degree from the University of Southampton, Southampton, U.K., in 1999, where he also received the Ph.D. degree from the Optoelectronics Research Centre (ORC), in 2003.

$\mathrm{He}$ is currently a Senior Research Fellow at the ORC. His Ph.D. thesis investigated the optical properties of various photonic devices at a nanometer scale using an interferometric near-field technique. He was involved in research in the field of nanophotonics in the Department of Physics, University of Southampton, before returning to the ORC in 2006 . He is currently involved in the development of planar-integrated optical devices for a wide range of applications in telecommunications and detection.

Andrew S. Webb received the B.Sc. degree in physics and electronics from Keele University, Staffordshire, U.K., in 1998. He is currently working toward the Ph.D. degree at the University of Southampton, Southampton, U.K.

In 2001, he became a Silica Fiber Fabrication Engineer at the Optoelectronics Research Centre, University of Southampton. He has coauthored more than 30 publications and patents. His current research interests include developing novel modified chemical vapor deposition rare-earth-doped preform fabrication techniques, optical fiber drawing, and production of specialty optical fibers.

Mr. Webb is a member of the Institute of Engineering and Technology, a member of the Institute of Physics, and a student member of the Optical Society of America.

Lewis G. Carpenter received the B.E. degree (with first class) in electrical engineering from the University of Southampton, Southampton, U.K., in 2009

$\mathrm{He}$ is a Postgraduate Research Student at the Optoelectronics Research Centre, University of Southampton. His current studies explore physical micromachining techniques for integrated optics and micro-opto-mechanical systems.

Mr. Carpenter is the recipient of the National Grid Award for his dissertation.
Faisal Rafiq Mahamd Adikan received the Ph.D. degree from the Optoelectronics Research Centre, University of Southampton, Southampton, U.K., in 2007.

He is currently the Head of the Photonic Lightwave Circuit Group, Photonics Research Centre, University of Malaya, Kuala Lumpur, Malaysia, where he is involved in developing novel fabrication processes to incorporate optically active materials into a glass matrix. His Ph.D. research on flat fiber produced an international patent. He specializes in glass-based integrated optical devices for use in telecommunication and sensing applications. Apart from research and teaching, he is also an active member of his Faculty, holding a number of administrative posts including the Coordinator of the Telecommunication Engineering program. He has published more than 70 journal and conference papers on optics and engineering education. He was the Deputy Chair of two Technical Postgraduate Symposiums, and is the current Chairman for the Faculty of Engineering's Sports and Recreational Club. He also established the Junior Lecturer Forum, an informal platform for young staff members to discuss matters concerning career development.

Dr. Rafiq was the recipient of the Section Prize for the Best Engineering Research during presentations at the House of Commons (British Parliament) in 2006. He also received the International Society for Optical Engineers Educational Scholarship in optical science and engineering in 2004, and the Best Paper for Photonic Category during an international conference in 2003.

Peter G. R. Smith received the B.A. degree in physics and the D.Phil. degree in nonlinear optics from Oxford University, Oxford, U.K., in 1990 and 1993, respectively.

$\mathrm{He}$ is currently a Professor at the Optoelectronics Research Centre and in the Department of Electronics and Computer Science, University of Southampton. After a year spent as a Management Consultant, he joined the University of Southampton, where he currently leads a research group concentrating on the fabrication of optical devices using integrated optics technology, particularly on direct UV writing and precision machining of optical materials. He is also involved in research on periodic poling technology and nonlinear optics. He has more than 200 publications, 10 patents, and was the founder of two spin-outs from Southampton-Stratophase Ltd. in 2003 and Covesion Ltd., in 2009.

Jayanta K. Sahu received the Ph.D. degree in physics from the India Institute of Technology Kharagpur, Kharagpur, India, in 1997.

From 1997 to April 2000, he was a Research Engineer at the Royal Institute of Technology, Stockholm, Sweden. In May 2000, he joined the Optoelectronics Research Centre, University of Southampton, Southampton, U.K., where he is currently a Principal Research Fellow and leading the Fiber Fabrication Group. He has published more than 250 papers in scientific journals and conference proceedings, including many invited and postdeadline papers. His current research interests include optical materials, development of novel silica-based fiber structures, and cladding-pumped rare-earth-doped fiber lasers and amplifiers

Dr. Sahu was the recipient of the 2005 U.K. Research Council Robert's (Academic) Fellowship Award. He is a member of the Optical Society of America. 